\title{
INTRODUÇÃO À FÍSICO-QUÍMICA ORGÂNICA UTILIZANDO UM COLORÍMETRO ARTESANAL - UMA PRÁTICA INTERDISCIPLINAR
}

\author{
Valmir B. Silva e Elisa S. Orth* \\ Departamento de Química, Universidade Federal do Paraná, CP 19081, 81531-990 Curitiba - PR, Brasil
}

Recebido em 01/07/2016; aceito em 13/09/2016; publicado na web em 12/10/2016

\begin{abstract}
INTRODUCTION TO PHYSICAL ORGANIC CHEMISTRY USING A HOMEMADE COLORIMETER - AN INTERDISCIPLINARY EXPERIMENT. Herein, we propose an interdisciplinary experiment for undergraduate course that involves concepts of organic and physical chemistry. Firstly, we propose the synthesis of three nitrophenyl acetates, varying the substituent position. Afterwards, we describe the construction of a homemade colorimeter using inexpensive materials and LED as both emission and detection dispositives. Finally, the colorimeter is used to follow the nucleophilic acyl transfer reactions catalyzed by imidazole under pseudo-first order conditions for the reactions of p-nitrophenyl, o-nitrophenyl and m-nitrophenyl acetate with imidazole. The substituent effect is correlated to the reactivity of the acetates, one of the benchmarks of a physical organic chemical approach. The effect of imidazole concentration is also evaluated. The proposed experiment is interesting because the students can thoroughly understand how a spectrophotometer works and since each student can construct their own colorimeter, they don't have to rely on any equipment infrastructure. Additionally they can correlate two important areas: organic and physical chemistry by using a kinetic study in the understanding of organic reactions. Indeed, physical organic chemistry is a field of increasing interest specially for elucidating mechanisms and introducing students to this interdisciplinary approach is interesting since several undergraduate concepts are covered.
\end{abstract}

Keywords: homemade colorimeter; kinetic study, physical organic chemistry, substituent effect.

\section{INTRODUÇÃO}

A físico-química orgânica é a área que correlaciona estrutura e reatividade em reações orgânicas, aliando ferramentas da físico-química com a química orgânica. ${ }^{1}$ Essa interdisciplinaridade tem permeado importantes descobertas na elucidação mecanística de diversas reações, no entanto essa área é pouco explorada nos currículos de graduação. Cada vez mais, a abordagem de inter-relacionar áreas da química tem se mostrado uma promissora aliada didática, o que atrelado ao fato que a área da físico-química orgânica "renasceu" no século XX, após sua quase decadência, corrobora a importância de apresentar esse assunto aos alunos de graduação. ${ }^{2}$ Nesse sentido, nós apresentamos um experimento interdisciplinar focado para cursos de graduação, com o objetivo de introduzir a físico-química orgânica através de um estudo cinético referente ao efeito do substituinte em reações nucleofílicas de desacilação, utilizando um colorímetro artesanal.

Reações nucleofílicas de desacilação de ésteres constituem uma interessante classe de reações para uma abordagem físico-química orgânica, uma vez que a estrutura do éster e do nucleófilo afetam a velocidade da reação. Conforme ilustrado no Esquema 1, nessas reações o nucleófilo ataca o centro eletrofílico do éster, podendo passar por um intermediário ou estado de transição (ET) tetraédrico, quando segue um mecanismo associativo ou concertado, respectivamente. Em seguida, ocorre a eliminação da base mais fraca ligada ao intermediário ou ET. ${ }^{3}$ Dessa forma, o $\mathrm{p} K_{\mathrm{a}}$ dos grupos abandonadores são bons indícios da reatividade de ésteres nessa classe de reações. Isso é, em geral, quanto menor o $\mathrm{p} K_{\mathrm{a}}$ do ácido conjugado do grupo abandonador, melhor grupo abandonador é. No caso do Esquema 1, como ROH tem $\mathrm{p} K_{\mathrm{a}}$ menor que $\mathrm{RH}\left(\mathrm{p} K_{\mathrm{a}}{ }^{\mathrm{ROH}} \sim 16 ; \mathrm{p} K_{\mathrm{a}}{ }^{\mathrm{RH}} \sim 50\right),{ }^{4}$ espera-se a saída de $\mathrm{RO}^{-}$em contraste a $\mathrm{R}^{-}$. Por outro lado, quando os valores de $\mathrm{p} K_{\mathrm{a}}$ dos grupos abandonadores são similares outros fatores devem ser levados em consideração para diferenciar a reatividade dos substratos, por exemplo, o efeito indutivo dos grupos próximos à carbonila.

\footnotetext{
*e-mail: elisaorth@ufpr.br
}

$\mathrm{O}$ experimento aqui proposto busca avaliar a reatividade dos acetatos de $p$-nitrofenila (APNF), $o$-nitrofenila (AONF) e $m$-nitrofenila (AMNF) nas reações de catálise nucleofílica mediadas por imidazol. O imidazol é conhecido por ser um catalisador nucleofílico nas reações com os ésteres de nitrofenila, sendo reativo apenas em sua forma neutra, onde o imidazol ataca o carbono da carbonila formando um intermediário acilado, seguido da sua hidrólise e regeneração do imidazol (Esquema 2). ${ }^{5,6} \mathrm{O}$ intermediário tetraédrico apontado no Esquema 1 previsto pelo mecanismo associativo foi desconsiderado no Esquema 2, visto sua difícil detecção. De fato, para a reação do imidazol com o APNF, é conhecido que a reação ocorre por um mecanismo de adição-eliminação (associativo) com a formação do intermediário tetraédrico seguido do intermediário acilado. Todavia, o intermediário tetraédrico é instável e rapidamente hidrolisado, não sendo possível observar sua existência pelos estudos cinéticos. Já o intermediário acilado já foi observado por UV-vis sendo possível acompanhar sua cinética de formação e decomposição. ${ }^{6,7}$

Assim, além de acelerar a velocidade da reação, o imidazol não é consumido, se comportando como um autêntico catalisador nucleofílico. O propósito do imidazol é promover a clivagem dos acetatos de nitrofenila para seus produtos nitrofenol e acetato, que são os mesmos produtos da hidrólise espontânea desses ésteres. No entanto, essa hidrólise é desfavorável e sua reação é conhecidamente lenta em água $\mathrm{pH} \sim 7$. Como o imidazol é um melhor nucleófilo que a água, a formação do intermediário com imidazol (Esquema 2) é mais favorável do que o respectivo com água (i.e. acetato). Em seguida, o acetato é facilmente obtido pela hidrólise do instável intermediário imidazólico, em contraste com a hidrólise do éster, visto o imidazol ser um bom grupo abandonador. Assim, os produtos da hidrólise dos ésteres podem ser obtidos utilizando o imidazol como catalisador nucleofílico. Vale ressaltar que o imidazol é um heterociclo bastante versátil com $\mathrm{p} K_{\mathrm{a}}$ do ácido conjugado $\sim 7$ e pode atuar como ácido, base ou nucleófilo. Como nucleófilo, apenas um nitrogênio do imidazol é nucleofílico, visto que o par de elétrons do outro nitrogênio está comprometido com a ressonância do anel (análogo ao pirrol). ${ }^{3}$ 


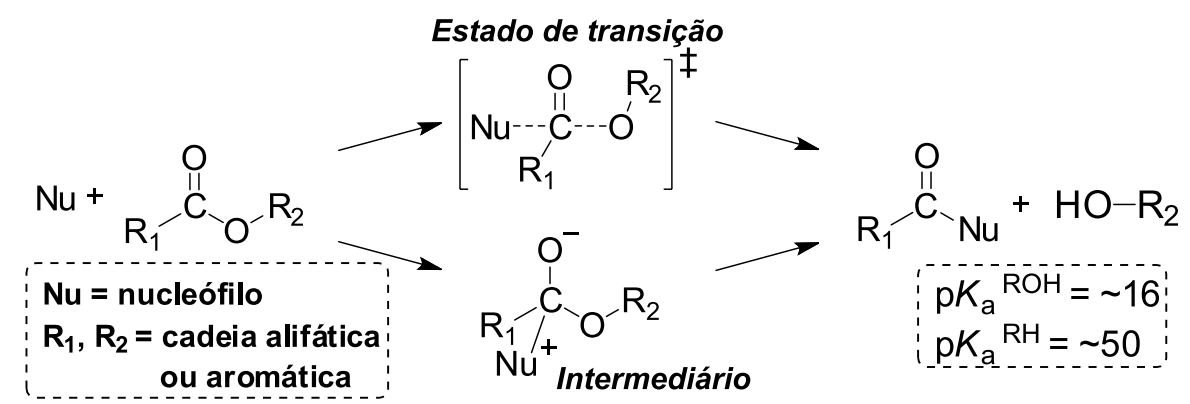

Esquema 1. Esquema geral para reações de adição seguido de eliminação em ésteres<smiles>O=C(C=CC[n+]1cc[nH]c1)Oc1ccc([N+](=O)[O-])cc1</smiles>

Intermediário<smiles>CC(=O)[n+]1cc[nH]c1Oc1ccc([N+](=O)[O-])cc1</smiles><smiles>CC(=O)O</smiles>

Esquema 2. Mecanismo para a reação dos ésteres de nitrofenila catalisado por imidazol

Com relação aos grupos abandonadores, nas reações propostas no Esquema 2 (primeira etapa para formação do intermediário), o $m$-nitrofenol tem basicidade acentuadamente maior $\left(\mathrm{MNF} \mathrm{p} K_{\mathrm{a}}=\right.$ $8,10)^{8}$ ao passo que o $p$-nitrofenol (PNF) e $o$-nitrofenol (ONF) tem basicidade semelhante (PNF $\mathrm{p} K_{\mathrm{a}}=7,15$ e ONF $\left.\mathrm{p} K_{\mathrm{a}}=7,23\right) .{ }^{9}$ De tal modo, comparado ao $\mathrm{CH}_{3}$, os nitrofenóis são melhores grupos abandonadores, portanto, espera-se que os produtos MNF, PNF e ONF sejam formados. Assim, AMNF, APNF e AONF são mais reativos que o acetato de fenila, visto que fenol tem $\mathrm{p} K_{\mathrm{a}}=9,{ }^{8}$ portanto sua saída é menos favorecida que o PNF, ONF ou MNF. O efeito retirador dos grupos nitro (por efeito indutivo e/ou ressonante) aumenta a eletroficilidade do éster, tornando-o mais suscetível ao ataque nucleofílico. ${ }^{10}$ Nesse contexto, espera-se que o AMNF seja menos reativo que o AONF e APNF, por seu grupo abandonador (MNF) possuir um $\mathrm{p} K_{\mathrm{a}}$ maior. Todavia, quando comparamos o PNF e ONF, seus $\mathrm{p} K_{\mathrm{a}}$ 's semelhantes dificultam uma distinção clara do melhor grupo abandonador, mas os resultados claramente mostram que APNF e AONF tem reatividades distintas. Para explicar isso, deve-se considerar o efeito do grupo retirador na posição orto, que tem efeito indutivo (além de efeito ressonante), que facilita a desacilação, deixando o centro eletrofílico mais reativo. ${ }^{5}$ Esses conceitos de reatividades são fundamentais para entender reações orgânicas e são os pilares da físico-química orgânica

Os aspectos abordados acima podem ser avaliados por estudos cinéticos com métodos experimentais que acompanham a concentração (de reagentes, intermediários e/ou produtos) em função do tempo. Para isso, são frequentemente utilizados pHmetros, condutivímetros, cromatógrafos ou espectrofotômetros. O uso de espectrofotômetros UV-VIS é bastante conhecido, para os quais existem diversos experimentos descritos em livros didáticos e revistas especializadas, que em geral exigem um mínimo de infraestrutura de equipamentos. ${ }^{11-14}$ Os espectrofotômetros são por muitas vezes tratados como "caixas-mágicas" onde uma amostra é inserida e um resultado é obtido sem que fique claro como o equipamento gerou o resultado. ${ }^{15}$ Assim, a construção de equipamentos de baixo-custo pode fazer uma dupla-função no processo de ensino e aprendizagem. Primeiro, elucidando como funciona um equipamento "por dentro", permitindo a discussão de aspectos técnicos sobre como gerar e melhorar a qualidade dos dados obtidos. Segundo, como ferramenta de acesso a esses equipamentos que frequentemente não existem, não funcionam ou têm seu uso restringido devido à grande quantidade de estudantes, uma vez que em geral os laboratórios de ensino possuem 1-2 espectrofotômetro(s).

Dessa forma, o objetivo desse trabalho é apresentar um experimento interdisciplinar para cursos de graduação em Química com objetivo de introduzir a físico-química orgânica através do estudo cinético de reações a fim de avaliar o efeito de substituinte na reatividade. Nesse experimento, o aluno utilizará várias áreas da química, i.e., química orgânica, físico-química e físico-química orgânica, pois irá sintetizar os ésteres, construir seu próprio colorímetro artesanal de baixo custo utilizando diodos emissores de luz (LED) para acompanhar as reações, realizar os estudos cinéticos e por fim interpretar os resultados associando reatividade com estrutura. Assim, o aluno aprenderá diversos conceitos: (i) funcionamento de espectrofotômetros; (ii) estudo cinético de reações sob condições de pseudo-primeira ordem; (iii) elucidação mecanística de reações nucleofílicas de desacilação e efeito do substituinte; e (iv) catálise nucleofílica. $\mathrm{O}$ experimento proposto foi aplicado com sucesso em uma turma de graduação em Química do quarto ano.

\section{PARTE EXPERIMENTAL}

O roteiro proposto para os alunos está apresentado no material suplementar, divididos em três partes que foram planejados para três aulas com duração de duas horas cada: (i) síntese dos acetatos de nitrofenila; (ii) construção do colorímetro artesanal e (iii) estudo físico-químico orgânico. Abaixo está descrito resumidamente os reagentes e materiais necessários, as sínteses, a construção do colorímetro artesanal, a validação do colorímetro através de uma curva de calibração e, por fim, o estudo cinético de reações de desacilação com imidazol utilizando o colorímetro.

\section{Reagentes e materiais necessários}

Os materiais necessários incluem LED alto brilho de cor azul, fios com conector macho e fêmea para protoboard, fonte de energia 
$(5 \mathrm{v} \sim 9 \mathrm{v})$, espuma rígida, estilete, resistores (100 ohm), fita adesiva, cubetas de acrílico, frasco com tampa e rosca, barra magnética, funil de separação, provetas, micropipetas automáticas ( $20 \mu \mathrm{L})$, pipetas volumétricas e graduadas $(5 \mathrm{~mL})$ e cronômetro. Os reagentes necessários são PNF, ONF, MNF, anidrido acético, perclorato de zinco hexahidratado, sulfato de sódio, hidróxido de sódio, imidazol, acetonitrila, éter etílico e água destilada. Como os alunos irão construir seu próprio colorímetro ("equipamento"), os equipamentos necessários para o experimento proposto serão o multímetro para medidas do colorímetro, agitador magnético, evaporador rotativo e medidor de ponto de fusão para a caracterização dos ésteres.

\section{Síntese dos ésteres}

Os ésteres APNF, AMNF e AONF foram sintetizados de acordo com a metodologia descrita na literatura. ${ }^{16}$ Em um frasco com tampa e rosca foram adicionados $2,5 \mathrm{mmol}$ de anidrido acético (de preferência destilado), 2,5 mmol de PNF, MNF ou ONF (para síntese do APNF, AMNF e AONF, respectivamente) e $0,025 \mathrm{mmol}$ de perclorato de zinco hexahidratado. A solução foi deixada sob agitação magnética por 10 minutos e em seguida foram adicionados $10 \mathrm{~mL}$ de água destilada. Posteriormente, realizou-se três extrações com $15 \mathrm{~mL}$ de éter etílico. A fase orgânica foi lavada (3x) com solução de hidróxido de sódio (125 $\left.\mathrm{mmol} \mathrm{L}^{-1}\right)$ para a neutralização do ácido acético presente. A fase orgânica foi seca com sulfato de sódio, filtrada e concentrada em um evaporador rotativo (de preferência sob pressão reduzida). Os sólidos obtidos possuem cor amarelo pálido e seu ponto de fusão (PF) foi determinado em um equipamento de ponto de fusão digital. Para APNF PF $=77^{\circ}-79^{\circ} \mathrm{C}$ (lit: $\left.78^{\circ}-79^{\circ} \mathrm{C}\right),{ }^{17} \mathrm{AONF} \mathrm{PF}=34^{\circ}-37^{\circ} \mathrm{C}$ (lit: $\left.38^{\circ} \mathrm{C}\right)^{18}$ e AMNF $\mathrm{PF}=49^{\circ}-52^{\circ} \mathrm{C}$ (lit: $\left.53^{\circ}-54^{\circ} \mathrm{C}\right) .^{19}$

\section{Construção do colorímetro}

Inicialmente, para construção do colorímetro deve-se montar um suporte, no qual foi utilizada uma peça de espuma rígida, porém, outros materiais podem ser utilizados. O suporte terá os LEDs emissor/receptor e o encaixe para apoiar a cubeta. A sequência de cortes na espuma, juntamente às dimensões do suporte, está apresentada na Figura 1.
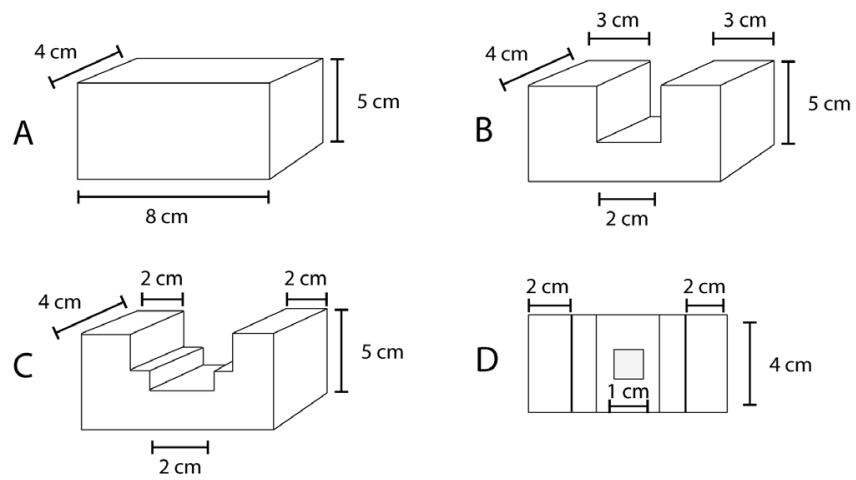

Figura 1. Montagem do suporte do colorímetro. $A=$ Bloco retangular inicial, $B=$ Corte central, $C=$ Segundo corte central para acomodação dos LEDs e $D=$ Vista de cima do suporte e corte quadrado para a acomodação da cubeta

Para a montagem da fonte de radiação são necessários 1 LED azul, dois fios para protoboard com entradas fêmeas, uma fonte de energia $(5 \mathrm{v} \sim 9 \mathrm{v})$ e um resistor $(100 \Omega)$. O LED apresenta uma "perna" mais comprida que a outra, referente ao polo positivo. Os fios, o resistor e o LED podem ser obtidos em lojas especializadas ou em lixo eletrônico, barateando ainda mais a montagem. A fonte de energia utilizada foi um carregador de celular, porém carregadores de outros equipamentos como telefones sem-fio, balanças, impressoras ou baterias comuns podem ser utilizados. Com o suporte montado, o LED foi encaixado. Nas pernas do LED os fios foram conectados, onde no fio ligado ao polo positivo o resistor foi preso e por fim conectados à fonte de energia de acordo com a Figura 2. Para a montagem do detector foi utilizado outro LED azul, dois fios para protoboard com entradas fêmeas e um multímetro com pontas de prova na forma de "jacarés". O LED detector foi posicionado alinhadamente ao LED emissor, os fios conectados às pernas do LED e suas respectivas pontas foram conectadas às pontas de prova do multímetro. A Figura 2 apresenta uma foto do sistema construído e a disposição dos componentes.

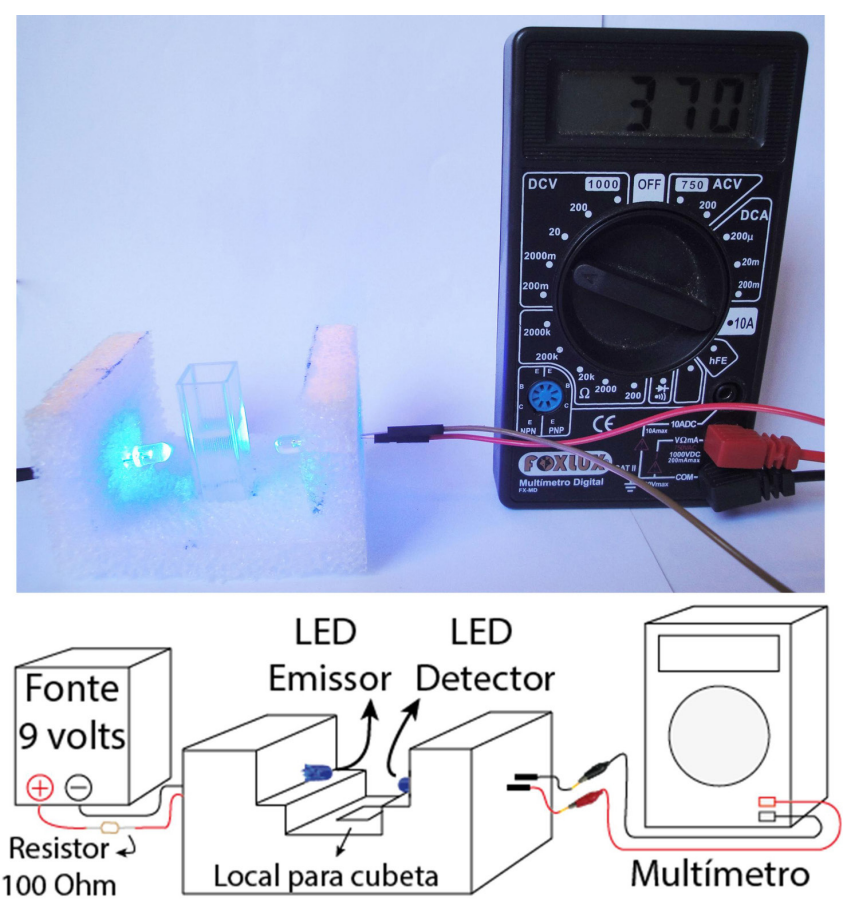

Figura 2. Foto do colorímetro construído e esquema da sua montagem

\section{Validação do colorímetro através de curva de calibração}

Para validar o uso do colorímetro construído, realizou-se uma curva de calibração com PNF para verificar a linearidade do perfil de absorbância (convertido a partir de dados da diferença de potencial, ddp, vide infra) vs concentração, bem como obter a absortividade molar e relacionar com a literatura. Inicialmente, mediu-se a ddp no LED detector utilizando o multímetro, com $100 \%$ de transmitância (colorímetro com a cubeta com $3 \mathrm{~mL}$ de água), ddp $\mathrm{dp}_{100 \%}$, e $0 \%$ de transmitância (objeto opaco bloqueando totalmente a passagem de

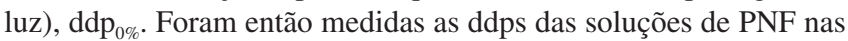
concentrações $1,0 \times 10^{-6}, 1,0 \times 10^{-5}, 3,0 \times 10^{-5}$ e $5,0 \times 10^{-5} \mathrm{~mol} \mathrm{~L}^{-1}$ em meio básico $(\mathrm{pH}=10)$. A medida de $\mathrm{ddp}_{100 \%}$ e ddp $\mathrm{dd}_{0 \%}$ é importante para a calibração do colorímetro, pois corresponde à máxima e mínima transmitância possível e também corrige a contribuição de eventual radiação externa. Uma calibração análoga é feita em alguns equipamentos comerciais, justificando a necessidade dessas medidas.

\section{Acompanhamento cinético}

Inicialmente mediu-se $\mathrm{ddp}_{100 \%}$ e ddp $0 \%$ conforme explicado acima. Em quatro cubetas, foram adicionadas $3 \mathrm{~mL}$ de cada solução imidazol

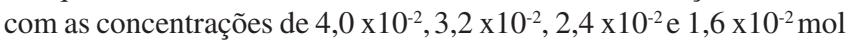
$\mathrm{L}^{-1}$. Cada reação iniciou com a adição de $20 \mu \mathrm{L}$ da solução estoque 
do acetato (APNF, AONF ou AMNF $5 \times 10^{-3} \mathrm{~mol} \mathrm{~L}^{-1} \mathrm{em}$ acetonitrila) e o cronômetro foi acionado. A ddp foi medida a partir do início da reação, com intervalos de 10 segundos nos primeiros 2 minutos e após com intervalo de 1 minuto, até a ddp ficar constante. Para fins de ilustração, as reações com imidazol mais concentrado $\left(4,0 \times 10^{-2}\right.$ mol L $\left.{ }^{-1}\right)$, i.e. mais rápidas, foram acompanhados por 4, 6 e 10 min para AONF, APNF e AMNF, respectivamente. Já nas reações com imidazol menos concentrado $\left(1,6 \times 10^{-2} \mathrm{~mol} \mathrm{~L}^{-1}\right)$, i.e. mais lentas, acompanhou-se por 9, 13 e 17 min para AONF, APNF e AMNF, respectivamente. No final, foram acompanhadas 12 reações diferentes. O material suplementar apresenta uma tabela completa como guia do que deve ser medido.

\section{RESULTADOS E DISCUSSÃO}

\section{Colorímetro: construção e validação}

O colorímetro tem o objetivo de substituir um espectrofotômetro comercial e para isso é necessário que ele seja capaz de, em essência, fazer as mesmas funções. Os espectrofotômetros comerciais tem como fonte de radiação lâmpadas de deutério e tungstênio e um prisma ou grade de difração para separar a radiação em seus comprimentos de onda constituintes. O equipamento aqui descrito é constituído por dois circuitos, um emissor de luz e outro receptor, funcionando como um detector. A Figura 3 mostra a disposição dos componentes para os dois circuitos. Os LEDs atuam como fonte de radiação e como seletor de comprimento de onda. Eles são construídos com materiais semicondutores e por isso apresentam band-gap definido em uma pequena faixa de comprimento de onda. Assim, a escolha da cor do LED para a montagem do colorímetro determina a faixa do comprimento de onda emitido na radiação luminosa. ${ }^{20}$ Para os nitrofenóis avaliados nesse experimento que apresentam seus máximos de absorção próximos a $400 \mathrm{~nm}$ (Figura 4B), o LED de cor azul, dentre as opções comerciais, se mostra como ideal pois seu máximo de emissão é aproximadamente 430 nm (linha azul tracejada mostrada na Figura 4B).

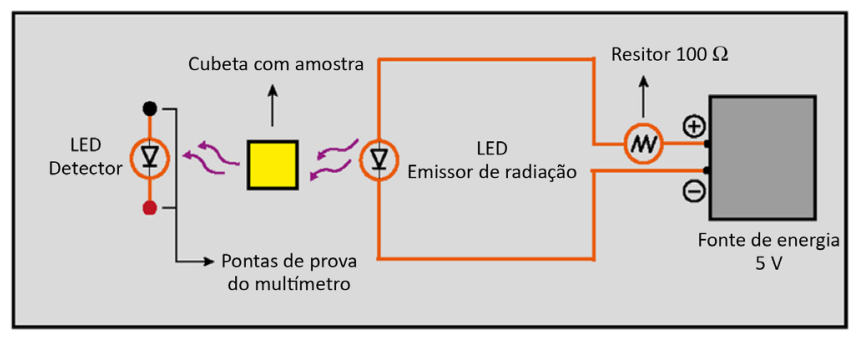

Figura 3. Esquema do colorímetro

O detector do colorímetro é constituído por um LED, sendo que o multímetro faz o papel de interface entre o equipamento e a geração dos dados. Os LEDs, quando submetidos a uma tensão, geram radiação e, por outro lado, quando a radiação incide sobre eles ocorre o inverso, a radiação é transformada em tensão, podendo ser medida com o auxílio de um multímetro. Dessa forma, quando colocamos uma substância que absorve no comprimento de onda do LED emissor, parte da radiação é absorvida e a quantidade de luz que passa pela cubeta decai, diminuindo a tensão no LED detector. Assim, podemos relacionar a quantidade de luz que sai do LED emissor com a ddp gerada no LED detector. Podemos ainda relacionar a ddp medida com absorbância pela Equação 1 e 2, onde I = radiação transmitida, $\mathrm{I}_{0}=$ radiação da fonte, $\operatorname{ddp}(\mathrm{a})=\mathrm{ddp}$ da amostra, ddp $_{0 \%}$ $=$ ddp medida com $0 \%$ de transmitância, ddp $_{100 \%}=$ ddp medida com $100 \%$ de transmitância, $\mathrm{A}=$ absorbância, $\mathcal{E}=$ absortividade molar, $\mathrm{c}=$ concentração e 1 = caminho ótico $($ em geral $1 \mathrm{~cm}) .{ }^{15}$ Assim, podemos obter perfis cinéticos clássicos de absorbância $v s$ tempo através de medidas de ddp em função do tempo.

$$
\begin{gathered}
\frac{I}{I_{0}}=\frac{d d p[a]-d d p_{0 \%}}{d d p_{100 \%}-d d p_{0 \%}} \\
-\log \frac{I}{I_{0}}=A=\varepsilon . c . . l
\end{gathered}
$$

Para evidenciar o perfil linear de absorbância vs concentração previstos pela Lei de Lambert-Beer (eq. 2) usando o colorímetro artesanal, como observado em espectrofotômetros usuais, foi construída uma curva de calibração para PNF. A Figura 4 mostra os espectros UV-Vis do PNF, ONF e MNF em meio básico (pH 10) obtidos utilizando um espectrofotômetro comercial (Perkin Elmer lambda 650), na qual a linha tracejada representa a emissão do LED azul. A Figura 4A apresenta a curva de calibração obtida com PNF utilizando o colorímetro artesanal, corroborando o comportamento esperado, com um bom coeficiente de correlação $\left(r^{2}=0,99\right)$ e mostrando que o colorímetro tem resposta linear na faixa de concentração estudada. $\mathrm{O}$ valor de absortividade molar obtido pelo coeficiente angular dessa reta $6447,54 \mathrm{~L} \mathrm{~mol}^{-1} \mathrm{~cm}^{-1}( \pm 144,47)$ é menor que os valores apresentados na literatura $\left(18380 \mathrm{~L} \mathrm{~mol}^{-1} \mathrm{~cm}^{-1}\right),{ }^{21}$ e já era esperado, uma vez que a radiação emitida pelo LED difere do comprimento de onda onde a absorção do PNF é máxima ( 405 nm). Vale ressaltar que as medidas foram feitas em $\mathrm{pH} 10$ para garantir que PNF estivesse desprotonado $\left(\mathrm{p} K_{\mathrm{a}} \sim 7,1\right)$. Isso porque a banda de absorção máxima é deslocada de $317 \mathrm{~nm}$ para $405 \mathrm{~nm}$ e a absortividade molar é maior para espécie aniônica, garantindo medidas de absorbância maiores, i.e. maior ddp, diminuindo o erro das medidas.

\section{Estudos cinéticos}

Com o colorímetro devidamente validado com a curva de calibração e calibrado (medições de $0 \%$ e $100 \%$ de transmitância), foi realizado o acompanhamento cinético das reações do imidazol com os acetatos APNF, AONF e AMNF. As reações apresentadas no Esquema 3 foram acompanhadas pelo aparecimento do produto PNF, ONF e MNF para APNF, AONF e AMNF, respectivamente.

As reações foram estudadas sob condições de pseudo-primeira ordem, pois as concentrações de imidazol foram muito maiores que as do éster. Assim, a lei de velocidade de segunda ordem (equação 3) se reduz a uma lei de primeira ordem (equação 4), em que v = velocidade, $\mathrm{k}_{2}=$ constante de velocidade de segunda ordem, [Éster] = concentração dos éster, [Imidazol] = concentração de imidazol e $\mathrm{k}_{\mathrm{obs}}=$ constante de velocidade observada de primeira ordem.

$$
\begin{gathered}
\mathrm{v}=\mathrm{k}_{2}[\text { Éster }][\text { Imidazol }] \\
\mathrm{v}=\mathrm{k}_{\mathrm{obs}}[\text { Éster }] \\
\mathrm{k}_{\mathrm{obs}}=\mathrm{k}_{2}[\text { Imidazol }] \\
{[P]_{t}=[P]_{\infty}\left(1-e^{-k_{b b s}}\right)} \\
A_{t}=A_{0}+\left(1-e^{-k_{o b s} t}\right)\left(A_{\infty}-A_{0}\right) \\
\ln \left(A_{\infty}-A_{t}\right)=\ln \left(A_{\infty}-A_{0}\right)-k_{o b s} t
\end{gathered}
$$

O estudo cinético proposto descreve o acompanhamento dos produtos fenolatos das reações do Esquema 3, sendo que a lei integrada para formação de produto para reação de primeira ordem é descrita pela equação 6 , onde $[\mathrm{P}]_{\mathrm{t}}=$ concentração do produto em determinado tempo, $[\mathrm{P}]_{\infty}=$ concentração do produto no infinito, $k_{\text {obs }}=$ constante de velocidade observada de primeira ordem e $\mathrm{t}=$ tempo. Para correlacionar a concentração de produto com a absorbância, combina-se a equação 6 com a lei de Lambert-Beer, levando à equação 7 , em que $\mathrm{A}^{\infty}=$ absorbância no infinito e $\mathrm{A}_{\mathrm{t}}=$ absorbância 
(A)

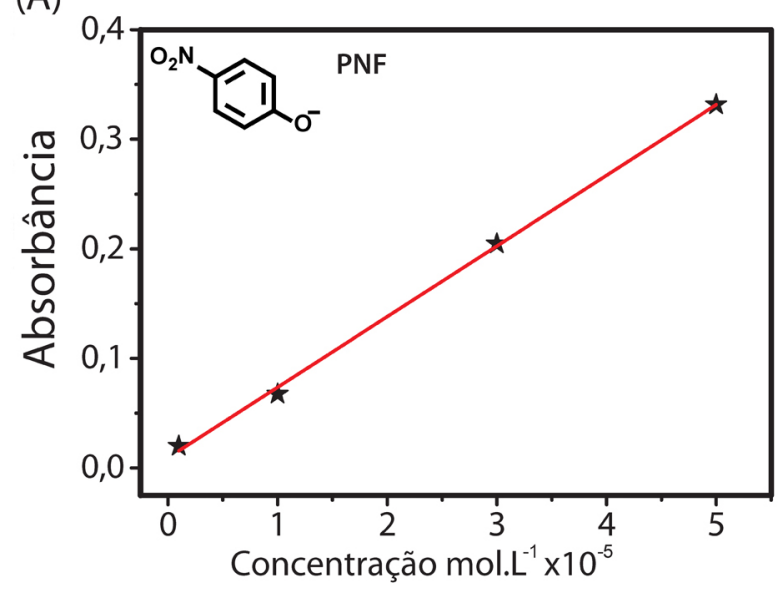

(B)

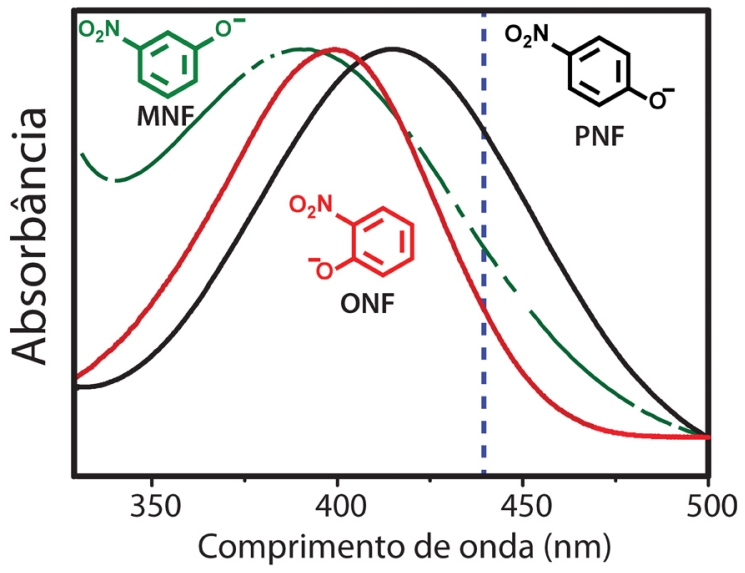

Figura 4. (A) Curva de calibração do PNF com o colorímetro e (B) Espectros do ONF, MNF e PNF. Linha azul tracejada corresponde a emissão do LED azul<smiles>CC(=O)Oc1ccc([N+](=O)[O-])cc1</smiles>

PNF<smiles></smiles>

AONF<smiles>CC(=O)Oc1ccccc1[N+](=O)[O-]</smiles><smiles>c1c[nH]cn1</smiles>

AMNF<smiles>CC(=O)Oc1cccc([N+](=O)[O-])c1</smiles><smiles>c1c[nH]cn1</smiles>

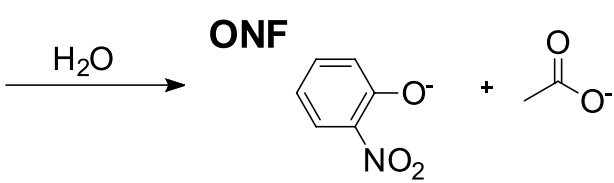

MNF<smiles>[N-]=[N+]([O-])c1cccc([O-])c1</smiles>

Esquema 3. Reações acompanhadas utilizando o colorímetro artesanal

em determinado tempo. Essa equação descreve os perfis de absorbância $v s$ tempo, tipicamente obtidos, apresentados na Figura 5. A equação 8 é a equação linearizada da equação 7, ou seja, ao plotar valores de $\ln \left(\mathrm{A}^{\infty}-\mathrm{A}_{\mathrm{t}}\right)$ e $\mathrm{t}$ no eixo y e $\mathrm{x}$, respectivamente, obtém-se um perfil linear que confirma o comportamento de primeira ordem e o coeficiente angular fornece $\mathrm{k}_{\mathrm{obs}}$. Para validar o uso do colorímetro artesanal para o acompanhamento cinético, tem-se, na Figura 5, o perfil cinético obtido utilizando o colorímetro artesanal e um equipamento comercial

(A)

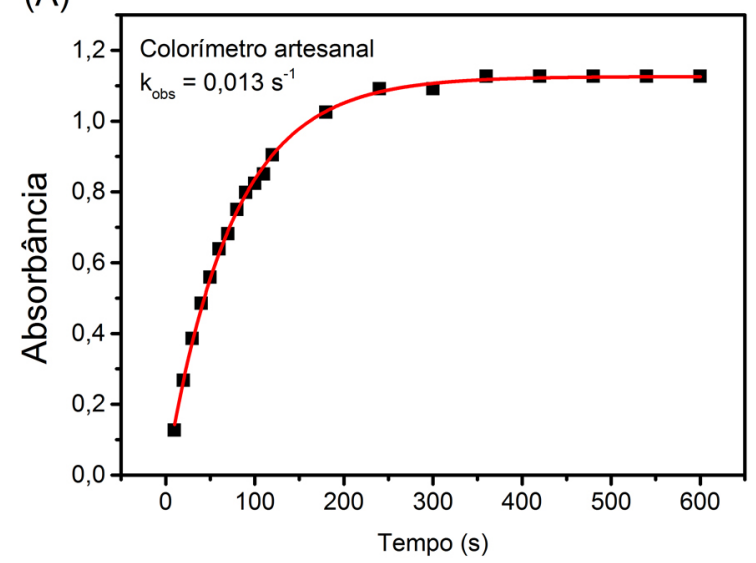

(espectrofotômetro UV-Vis) para reação do AONF com imidazol (0,015 mol L $\left.{ }^{-1}\right)$. Os valores de $k_{\text {obs }}$ obtidos para os perfis apresentados na Figura 5 foram similares (obtidos por ajuste não-linear, Eq. 7), evidenciando que o colorímetro artesanal pode substituir com segurança o espectrofotômetro comercial no acompanhamento cinético das reações.

Os perfis cinéticos obtidos utilizando o colorímetro artesanal para as reações propostas com diferentes concentrações de imidazol estão apresentados na Figura 6A, 6C e 6E, na forma linear (Eq. 8).

(B)

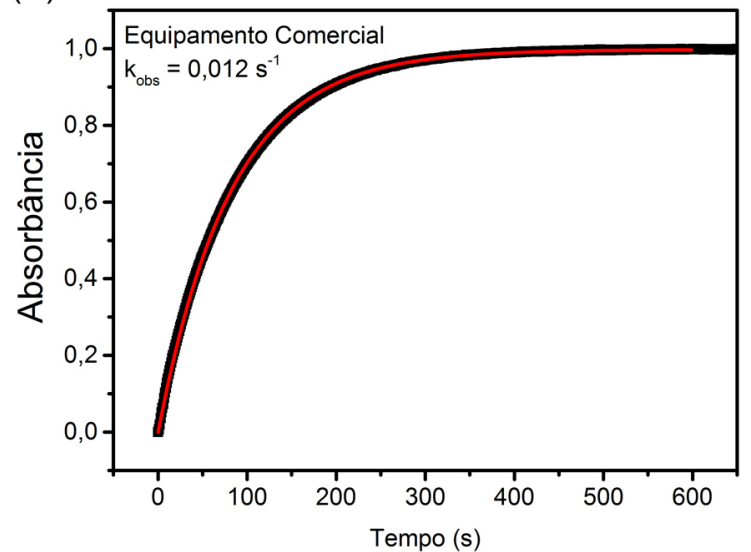

Figura 5. Dados de absorbância vs tempo para a reação do AONF com imidazol (0,015 mol $\left.\mathrm{L}^{-1}\right)$ acompanhadas com (A) colorímetro artesanal proposto e (B) espectrofotômetro comercial. Linhas vermelhas correspondem ao ajuste com a equação 7 
Observa-se que todas a reações seguiram um perfil de primeira ordem com relação ao produto fenolato (consequentemente também para o reagente acetato). $\mathrm{O}$ efeito da concentração do imidazol pode ser observado na Figura 6B, 6D e $6 \mathrm{~F}$ através de um perfil linear que está de acordo com a equação 5 , evidenciado que a reação é de primeira ordem em relação ao imidazol. Apesar do imidazol estar em excesso, aumentar a concentração dele, aumenta $\mathrm{k}_{\mathrm{obs}}$, tornando a reação mais rápida. Através dos perfis da Figura $6 \mathrm{~B}, 6 \mathrm{D}$ e $6 \mathrm{~F}$ foram obtidos as constantes de segunda ordem $\left(k_{2}\right)$ pelo coeficiente angular, que é a forma mais comum de representar constantes de velocidade nucleofílicas, pois pondera o efeito da concentração do nucleófilo (reagente em excesso).

(A)

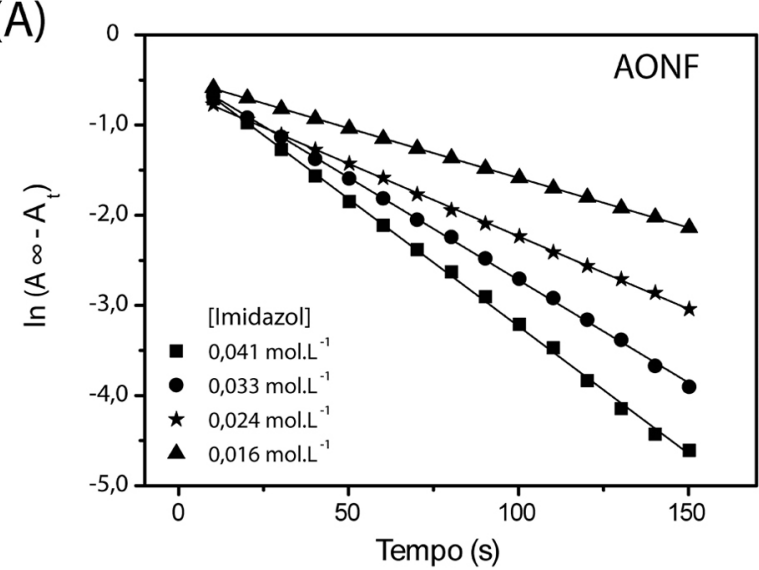

(C)

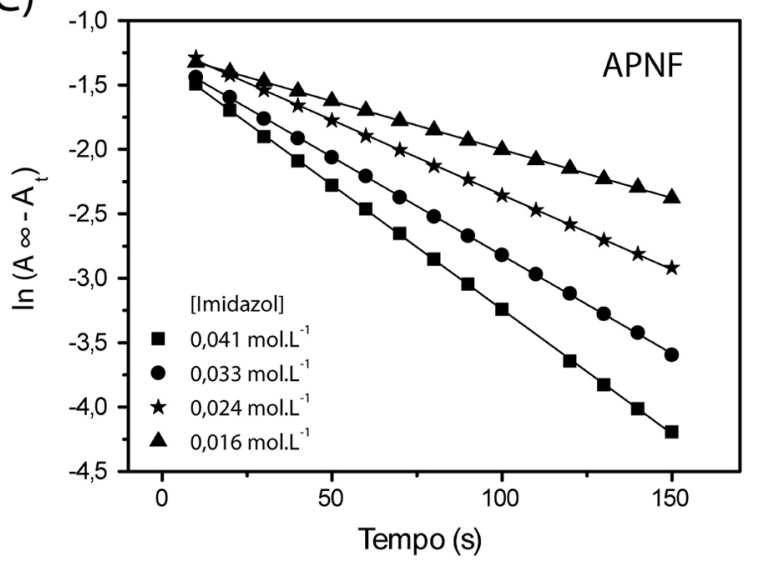

(E)

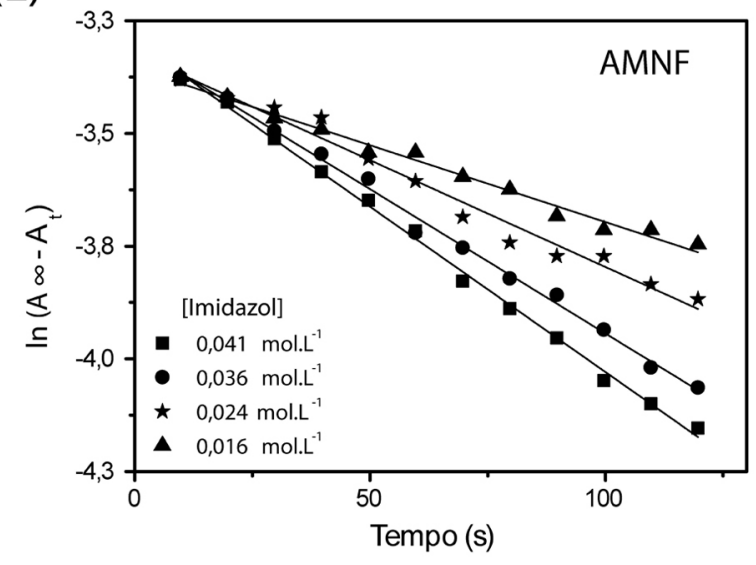

Vale ressaltar que, para as diferentes concentrações de imidazol,

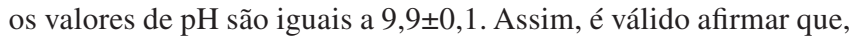
nas condições reacionais propostas, o imidazol está na forma neutra mais reativa (vide supra) e ainda, que a reação nucleofílica catalisada é a reação que está sendo monitorada. Isso porque dado a baixa concentração de íons hidroxila $\left(\sim 10^{-4} \mathrm{~mol} \mathrm{~L}^{-1}\right)$ aliada às pequenas constantes das reações espontânea e alcalina (e.g. para APNF $\mathrm{k}_{0}=$ $1,1 \times 10^{-5} \mathrm{~s}^{-1} \mathrm{e} \mathrm{k}_{\mathrm{OH}}=1,0 \times 10^{-4} \mathrm{~mol}^{-1} \mathrm{~L} \mathrm{~s}^{-1}$, respectivamente), ${ }^{22}$ assume-se que essas reações não contribuem significativamente para reação global nas condições reacionais. Isso é, o tempo de meia vida das reações com água ( $\mathrm{pH} 7$ ) e hidróxido ( $\mathrm{pH}$ 10) são 17,5 h e 26,7 meses, respectivamente, confirmando a pequena contribuição dessas

(B)

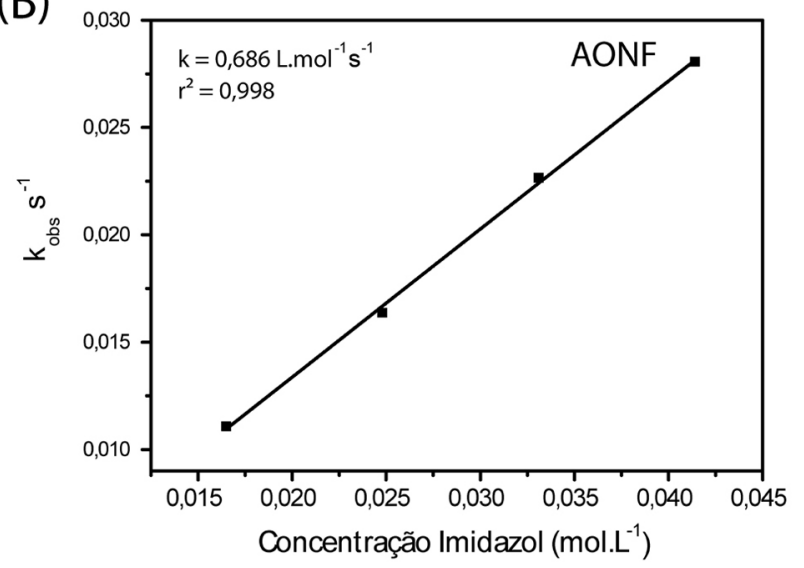

(D)

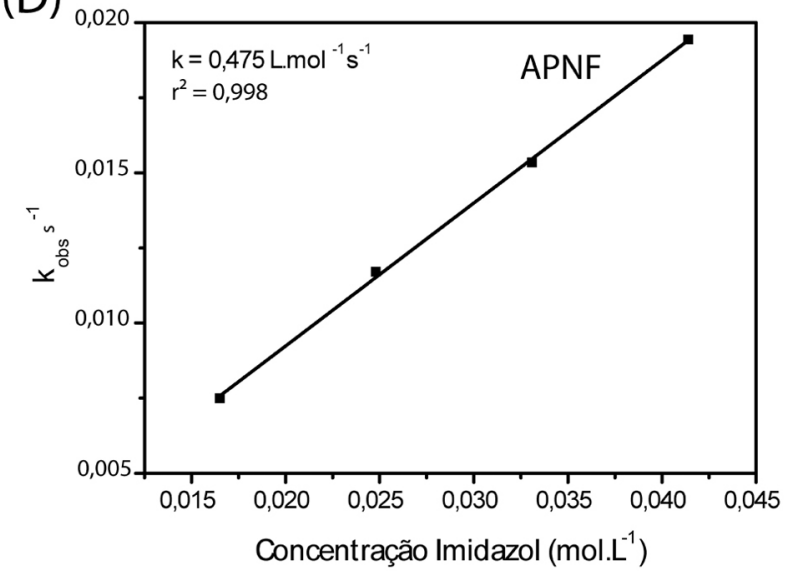

( $\mathrm{F})$

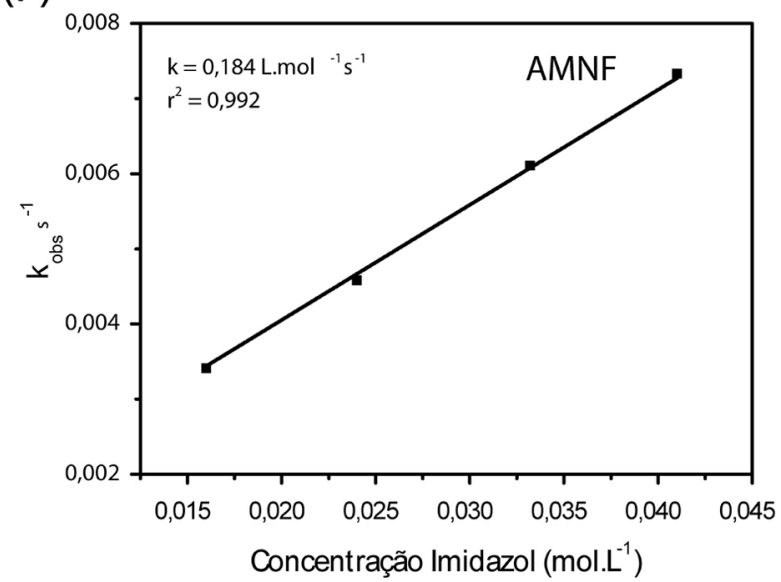

Figura 6. Perfis cinéticos para reações do (A) AONF (C) APNF e (E) AMNF com imidazol em diversas concentrações de imidazol e gráficos de $k_{\text {obs }}$ vs concentração do imidazol para (B) AONF (D) APNF e (F) AMNF 
Tabela 1. Dados obtidos para as reações acompanhadas utilizando o colorímetro artesanal

\begin{tabular}{|c|c|c|c|c|c|c|}
\hline \multirow{2}{*}{$\begin{array}{l}\text { [Imidazol] / } \\
10^{-2} \mathrm{~mol} \mathrm{~L}^{-1}\end{array}$} & \multicolumn{2}{|l|}{ APNF } & \multicolumn{2}{|l|}{ AONF } & \multicolumn{2}{|l|}{ AMNF } \\
\hline & $k_{\mathrm{obs}} / 10^{-3} \mathrm{~s}^{-1}$ & $\mathrm{k}_{2} / 10^{-1} \mathrm{~L} \mathrm{~mol}^{-1} \mathrm{~s}^{-1}$ & $k_{\mathrm{obs}} / 10^{-2} \mathrm{~s}^{-1}$ & $\mathrm{k}_{2} / 10^{-1} \mathrm{~L} \mathrm{~mol}^{-1} \mathrm{~s}^{-1}$ & $k_{\mathrm{obs}} / 10^{-3} \mathrm{~s}^{-1}$ & $\mathrm{k}_{2} / 10^{-1} \mathrm{~L} \mathrm{~mol}^{-1} \mathrm{~s}^{-1}$ \\
\hline 1,65 & $7,49( \pm 0,12)$ & \multirow{4}{*}{$4,75( \pm 0,09)$} & $1,10( \pm 0,04)$ & \multirow{4}{*}{$6,86( \pm 0,09)$} & $3,31( \pm 0,06)$ & \multirow{4}{*}{$1,84( \pm 0,08)$} \\
\hline 2,48 & $11,7( \pm 0,70)$ & & $1,63( \pm 0,11)$ & & $4,47( \pm 0,09)$ & \\
\hline 3,31 & $15,3( \pm 0,80)$ & & $2,26( \pm 0,14)$ & & $6,17( \pm 0,13)$ & \\
\hline 4,41 & $19,4( \pm 1,20)$ & & $2,80( \pm 0,24)$ & & $7,84( \pm 0,12)$ & \\
\hline
\end{tabular}

reações. Todos os resultados obtidos estão apresentados na Tabela 1.

Analisando os dados na Tabela 1 para os diferentes ésteres observa-se que as constantes $k_{2}$ seguem $A O N F>A P N F>A M N F\left(k_{\text {obs }}\right.$ segue mesma ordem nas diferentes concentrações) evidenciando o efeito do $\mathrm{p} K_{\mathrm{a}}$ do grupo de saída, onde AONF e APNF foram mais reativos que o AMNF, conforme esperado. De fato, a posição do substituinte nitro influencia de forma bastante acentuada a reatividade. No caso do AONF, tem-se um ataque nucleofilico mais suscetível devido ao efeito indutivo exercido sobre a carbonila, fato que é pouco perceptível para o APNF devido à maior distância entre o grupamento nitro e a carbonila. Quando a reatividade dos AONF e APNF é comparada ao acetato de fenila percebemos que os grupamentos nitro diminuem os $\mathrm{p} K_{\text {as }}$ dos grupos abandonadores (nitrofenóis, vide supra) e que essa diferença afeta majoritariamente a reatividade sendo o acetato de fenila bem menos reativo $\left(\mathrm{k}_{2}=0,0018 \mathrm{~L} \mathrm{~mol}^{-1} \mathrm{~s}^{-1}\right.$ para reação com imidazol ). ${ }^{23}$ A Figura 7 apresenta um resumo do estudo realizado, onde tem-se uma relação da reatividade $\left(\mathrm{k}_{2}\right)$ com a estrutura do éster e do seu grupo abandonador para levar a compreensão global dos efeitos. Assim, o grupo nitro é retirador de elétrons, o que torna o éster um melhor eletrófilo e diminui o $\mathrm{p} K_{\mathrm{a}}$ do grupo abandonador. Quanto maior esse $\mathrm{p} K_{\mathrm{a}}$, pior o grupo abandonador, portanto o AMNF é o menos reativo. No AMNF tem-se apenas o efeito indutivo do grupo nitro, pois o efeito ressonante na posição meta não afeta significativamente o éster. Já para o APNF, tem-se apenas o efeito retirador ressonante pois a contribuição do efeito indutivo é insignificante devido à distância do grupo retirador. Como o efeito ressonante é mais forte que o indutivo, APNF tem maior reatividade que AMNF. Por fim, o AONF se beneficia do efeito retirador ressonante e indutivo do grupo nitro, tornando-o mais reativo.

Dessa forma, o estudo cinético proposto permite relacionar reatividade (constante de velocidade) com estrutura (posição/natureza do substituinte) de forma bastante elegante, sendo estes aspectos os fundamentos da físico-química orgânica e o alicerce da elucidação

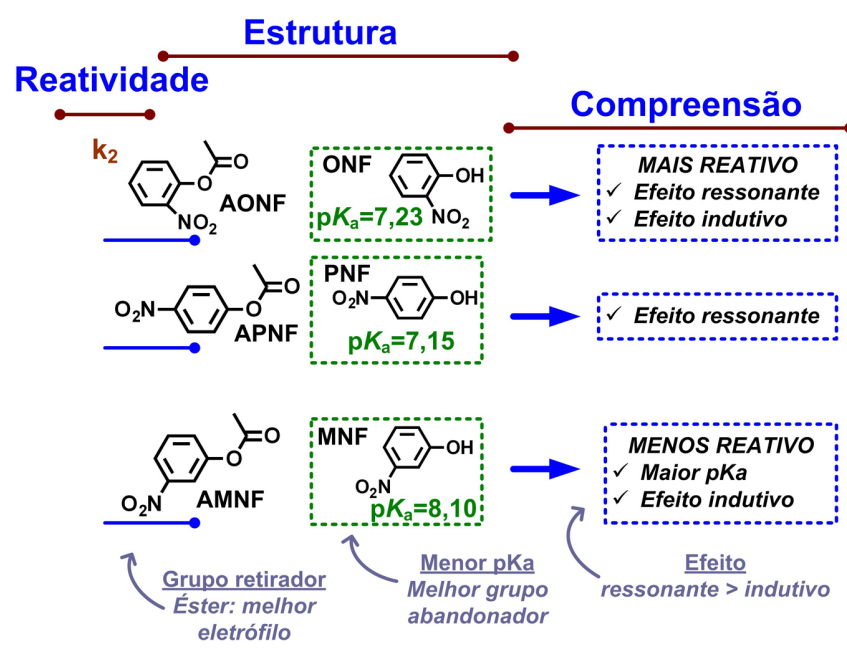

Figura 7. Resumo do estudo realizado e da compreensão dos efeitos avaliados mecanística das reações químicas. A compreensão de catálise nucleofílica também é fundamental e, através do mecanismo proposto, o imidazol efetivamente é um catalisador, pois reage e, ao término, é regenerado (Esquema 2). A regeneração do imidazol não pode ser distinguida cineticamente pelo experimento proposto, visto que ele está em excesso. No entanto, estudos anteriores confirmam esse caráter catalítico do imidazol, no qual foi possível acompanhar a regeneração do imidazol por ressonância magnética nuclear e espectrometria de massas. ${ }^{24}$ Esse aspecto mecanístico é fundamental para a discussão desse experimento e enriquece sua interdisciplinaridade, visto que esse papel catalítico do imidazol é conhecido em muitos sítios enzimáticos. ${ }^{6}$ Ainda, muitos conceitos cinéticos fundamentais foram abordados no experimento proposto. Um questionamento complementar que pode ser levantado: "qual seria a reatividade do acetato de 2,4-dinitrofenila em relação aos avaliados?". Nesse caso, o menor $\mathrm{p} K_{\mathrm{a}}$ do 2,4-dinitrofenol ( 4), devido ao efeito retirador do segundo grupo nitro, torna-o um melhor grupo abandonador que PNF, ONF e MNF. Da mesma forma, ao substituir o grupo nitro por grupos doadores como metila, tornam o éster menos reativo, visto que o $\mathrm{p} K_{\mathrm{a}}$ do fenol correspondente é maior. O efeito de substituinte também pode ser avaliado na reatividade do nucleófilo, no entanto, apresenta efeito inverso do eletrófilo. Espera-se que grupos retiradores (e.g. nitro) diminuam a nucleofilicidade do imidazol, enquanto grupos doadores (e.g. metila) aumentem. Dentro dos conceitos cinéticos, pode-se questionar qual seria o perfil esperado se acompanhássemos o consumo de reagente. De fato, seria esperado também um perfil de primeira ordem, mas devido ao comprimento de onda de emissão do LED azul ( 400 nm) não seria possível acompanhar o consumo de reagente com o colorímetro proposto, que ocorre $\sim 200 \mathrm{~nm}$.

\section{CONCLUSÃO}

O experimento proposto busca, inicialmente, através da construção do colorímetro, desmistificar como funciona um equipamento de espectrofotometria "por dentro", oferecendo ao professor a oportunidade de discutir diversos conceitos químicos como a lei de Lambert-Beer junto a aspectos técnicos referentes aos equipamentos de forma geral. Além disso, o experimento proporciona o acesso a equipamentos por um baixo custo, pois os comerciais, de forma geral, são caros e com quantidade limitada. Prevê-se que o custo do colorímetro artesanal seja $\sim \mathrm{R} \$ 10$, podendo ser menor se forem reutilizados carregadores de celular como fonte e ainda sem contabilizar multímetro, que em geral tem-se maior disponibilidade nos laboratórios de ensino. Ainda, os alunos aplicam conceitos da química orgânica para sintetizar os ésteres por rotas simples e rápidas. Finalmente, o estudo cinético busca a discussão de aspectos fundamentais da estrutura e reatividade de compostos orgânicos proporcionando um estudo interdisciplinar que se utiliza da cinética como ferramenta para a compreensão de mecanismos de reações orgânicas. Nessa parte, os alunos aplicam importantes fundamentos da cinética química como ordem de reação em relação ao diferentes reagentes, condições de pseudo-n-ordem e constantes de velocidades para correlacionar com a 
reatividade. $\mathrm{O}$ acompanhamento de produto também não é usual para alunos de graduação, visto que poucos livros didáticos apresentam a lei integrada para produtos, pois, em geral, só se avalia cinética em função do consumo de reagente. Ainda, eles aprendem o que é uma reação catalítica nucleofílica, promovida por muitas enzimas biológicas, mas muito difícil de mimetizar em reações "simples" para estudar em laboratório. $\mathrm{O}$ experimento aqui proposto foi executado com êxito em uma turma de $4^{\circ}$ ano do curso de química da UFPR, na qual os alunos demonstraram elevado interesse e participação, possivelmente devido à interdisciplinaridade do experimento que permite abordar e correlacionar diferentes áreas. Ainda, a abordagem físico-química orgânica foi novidade para maioria dos alunos, mas como eles já possuem o conhecimento teórico de química orgânica e físico-química necessários, eles conseguiram assimilar as ferramentas cinéticas propostas e sua importância.

\section{MATERIAL SUPLEMENTAR}

Os detalhes experimentais com fotos passo a passo para a montagem do colorímetro, roteiro proposto para o experimento e dados adicionais referentes aos estudos cinéticos estão descritos no material suplementar e estão disponíveis em http://quimicanova.sbq.org.br, na forma de arquivo PDF, com acesso livre.

\section{AGRADECIMENTOS}

CNPq, CAPES, L'Oréal-UNESCO-ABC, Fundação Araucária e UFPR.

\section{REFERÊNCIAS}

1. Anslyn, E. V.; Dougherty, D. A.; Modern Physical Organic Chemistry, University Science, 2006.
2. Thiesen, J. da S.; Rev. Bras. Educ. 2008, 13, 545.

3. Clayden, J.; Greeves, N.; Warren, S.; Organic Chemistry, Oxford University Press: Oxford, 2012.

4. Solomons, T. W. G.; Fryhle, C.; Organic Chemistry, John Wiley \& Sons, 2009.

5. Holmquist, B.; Bruice, T. C.; J. Am. Chem. Soc. 1969, 91, 2985.

6. Bender, M. L.; Turnquest, B. W.; J. Am. Chem. Soc. 1957, 79, 1652.

7. Kirsch, J. F.; Jencks, W. P.; J. Am. Chem. Soc. 1964, 86, 837.

8. Bouchard, G.; Carrupt, P.-A.; Testa, B.; Gobry, V.; Girault, H. H.; Chem. Eur. J. 2002, 8, 3478.

9. Mock, W. L.; Morsch, L. A.; Tetrahedron 2001, 57, 2957.

10. Jones, J.; Core Carbonyl Chemistry, Oxford University Press, 1997.

11. Burgess, A. E.; Davidson, J. C.; J. Chem. Educ. 2014, 91, 300.

12. Dimmel, D. R.; McKinney, M. A.; J. Chem. Educ. 1972, 49, 373.

13. Garland, C. W.; Nibler, J. W.; Shoemaker, D. P.; Experiments in physical chemistry, McGraw-Hill Higher Education: Boston, 2009

14. Klotz, I. M.; J. Chem. Educ. 1945, 22, 328.

15. Tavener, S. J.; Thomas-Oates, J. E.; Educ. Chem. 2007, 44, 151.

16. Shivani; Gulhane, R.; Chakraborti, A. K.; J. Mol. Catal. A: Chem. 2007, 264, 208.

17. Ghosh, U.; Ganessunker, D.; Sattigeri, V. J.; Carlson, K. E.; Mortensen, D. J.; Katzenellenbogen, B. S.; Katzenellenbogen, J. A.; Bioorg. Med. Chem. 2003, 11, 629.

18. Stelmach, H.; Connors, K. A.; J. Am. Chem. Soc. 1970, 92, 863.

19. Williams, A.; Naylor, R. A.; J. Chem. Soc. B 1971, 1967.

20. Wagner, E. P.; J. Chem. Educ. 2016, 93, 1289.

21. Bowers, G. N.; McComb, R. B.; Christensen, R. G.; Schaffer, R.; Clin. Chem. 1980, 26, 724.

22. Goren, H. J.; Fridkin, M.; Eur. J. Biochem. 1974, 41, 263.

23. Bruice, T. C.; Schmir, G. L.; J. Am. Chem. Soc. 1957, 79, 1663.

24. Orth, E. S.; Almeida, T. G.; Silva, V. B.; Oliveira, A. R. M.; Ocampos, F. M. M.; Barison, A.; J. Mol. Catal. A: Chem. 2015, 403, 93. 